PROCEEDINGS OF THE

AMERICAN MATHEMATICAL SOCIETY

Volume 127, Number 9, Pages 2643-2651

S 0002-9939(99)05271-5

Article electronically published on May 4, 1999

\title{
WEIGHTED INEQUALITIES FOR ITERATED CONVOLUTIONS
}

\author{
KENNETH F. ANDERSEN
}

(Communicated by Frederick W. Gehring)

\begin{abstract}
Given a fixed exponent $p, 1 \leq p<\infty$, and suitable nonnegative weight functions $v_{j}, j=1, \ldots, m$, an optimal associated weight function $\omega_{m}$ is constructed for which the iterated convolution product satisfies

$$
\int_{0}^{\infty}\left|\left[\prod_{j=1}^{m} * F_{j}\right](x)\right|^{p} \frac{d x}{\omega_{m}(x)} \leq \prod_{j=1}^{m} \int_{0}^{\infty}\left|F_{j}(t)\right|^{p} \frac{d t}{v_{j}(t)}
$$

for all complex valued measurable functions $F_{j}$ with $\int_{0}^{\infty}\left|F_{j}(t)\right|^{p} d t / v_{j}(t)<\infty$. Here $\left[\prod_{j=1}^{2} * F_{j}\right](x)=\left[F_{1} * F_{2}\right](x)=\int_{0}^{x} F_{1}(t) F_{2}(x-t) d t$ and for each $m>2$, $\prod_{j=1}^{m} * F_{j}=\left[\prod_{j=1}^{m-1} * F_{j}\right] * F_{m}$. Analogous results are given when $R^{+}=(0, \infty)$ is replaced by $R^{n}$ and also when the convolution $F_{1} * F_{2}$ on $R^{+}$is taken instead to be $\int_{0}^{\infty} F(t) G(x / t) d t / t$. The extremal functions are also discussed.
\end{abstract}

\section{INTRODUCTION}

It is well known that the convolution $\left(F_{1} * F_{2}\right)(x)=\int_{0}^{x} F_{1}(t) F_{2}(x-t) d t$ of Lebesgue integrable functions $F_{1}, F_{2} \in L\left(R^{+}\right)$belongs to $L\left(R^{+}\right)$, and more generally, if $1 \leq p<\infty$ the convolution of $F_{1} \in L\left(R^{+}\right)$and $F_{2} \in L^{p}\left(R^{+}\right)$is also an $L^{p}\left(R^{+}\right)$function. Indeed, Young's Theorem asserts that

$$
\left\|F_{1} * F_{2}\right\|_{L^{p}\left(R^{+}\right)} \leq\left\|F_{1}\right\|_{L\left(R^{+}\right)}\left\|F_{2}\right\|_{L^{p}\left(R^{+}\right)} .
$$

However, the convolution of two $L^{p}\left(R^{+}\right)$functions need not belong to $L^{p}\left(R^{+}\right)$. If the ambient space $\Omega=R^{+}=(0, \infty)$ is replaced by $R^{n}$, the convolution $\left(F_{1} *\right.$ $\left.F_{2}\right)(x)=\int_{R^{n}} F_{1}(t) F_{2}(x-t) d t$ satisfies the analogue of (1.1), but for $F_{1}, F_{2} \in$ $L^{p}\left(R^{n}\right)$ the convolution need not exist, let alone belong to $L^{p}\left(R^{n}\right)$. The purpose of this paper is to determine, when $\Omega=R^{+}$or $R^{n}$, conditions on the measures $\mu_{j}$, $j=1,2$, on $\Omega$ which ensure that $F_{1} * F_{2}$ exists whenever $F_{j} \in L^{p}\left(\Omega, \mu_{j}\right)$ and to construct an associated measure $\nu$ on $\Omega$ so that $F_{1} * F_{2} \in L^{p}(\Omega, \nu)$ and satisfies a weighted substitute for (1.1), namely

$$
\left\|F_{1} * F_{2}\right\|_{L^{p}(\Omega, \nu)} \leq\left\|F_{1}\right\|_{L^{p}\left(\Omega, \mu_{1}\right)}\left\|F_{2}\right\|_{L^{p}\left(\Omega, \mu_{2}\right)} .
$$

In fact, we consider more general inequalities for the iterated convolution products $\prod_{j=1}^{m} * F_{j}$ defined by $\prod_{j=1}^{2} * F_{j}=F_{1} * F_{2}$ and $\prod_{j=1}^{m} * F_{j}=\left[\prod_{j=1}^{m-1} * F_{j}\right] * F_{m}$ for $m>2$.

Received by the editors June 24, 1997.

1991 Mathematics Subject Classification. Primary 26D15, 44A35, 42A85; Secondary 26D10.

Key words and phrases. Convolution, weights, inequalities.

This research was supported in part by NSERC research grant \#OGP-8185.

(C)1999 American Mathematical Society 
Our main result is the following theorem which generalizes a recent result of Cwikel and Kerman [2] and certain earlier results of Saitoh [4] and Burbea [1].

Theorem 1. Let $1 \leq p<\infty, 1 / p+1 / p^{\prime}=1$, and let $m \geq 2$ be an integer. Suppose $v_{j}, j=1, \ldots, m$, are nonnegative measurable functions on $R^{+}$with $v_{j}^{1 / p} \in L^{p^{\prime}}(0, R)$ for every $R>0$. Define $\omega_{1}=v_{1}$, and set

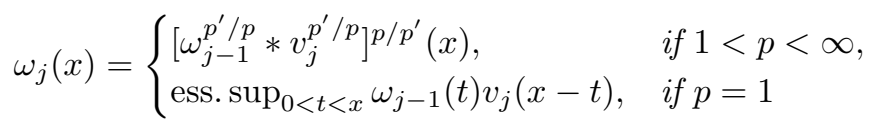

for $j=2, \ldots, m$. Then

$$
\int_{R^{+}}\left|\left[\prod_{j=1}^{m} * F_{j}\right](x)\right|^{p} \frac{d x}{\omega_{m}(x)} \leq \prod_{j=1}^{m} \int_{R^{+}}\left|F_{j}(t)\right|^{p} \frac{d t}{v_{j}(t)}
$$

for all complex valued measurable functions $F_{j}$ with $\int_{R^{+}}\left|F_{j}(t)\right|^{p} \frac{d t}{v_{j}(t)}<\infty, j=$ $1, \ldots, m$.

If $1<p<\infty$, equality holds in (1.2) if

$$
F_{j}(t)=c_{j} e^{\alpha t+i \beta t} v_{j}^{p^{\prime} / p}(t) \quad \text { a.e. }
$$

for complex constants $c_{j}$ and real numbers $\alpha, \beta$ such that $\int_{R^{+}} e^{p \alpha t} v_{j}^{p^{\prime} / p}(t) d t<\infty$, $j=1, \ldots, m$. Conversely, if $v_{j}(t)>0$ on an open interval $I_{j}$ and $v_{j}(t)=0$ on $R^{+} \backslash I_{j}, j=1, \ldots, m$, and equality holds in (1.2), then $F_{j}, j=1, \ldots, m$, is given by (1.3) unless $F_{j}(t)=0$ a.e. for some $j$.

For $r, s>0$ the Beta function $B(r, s)=\int_{0}^{1} y^{r-1}(1-y)^{s-1} d y=\Gamma(r) \Gamma(s) / \Gamma(r+s)$ shows that

$$
\left[t^{r-1} * t^{s-1}\right](x)=\frac{\Gamma(r) \Gamma(s)}{\Gamma(r+s)} x^{r+s-1}, \quad x>0,
$$

and hence, if $1<p<\infty, q_{j}>0$ and $v_{j}(t)=t^{\left(q_{j}-1\right)(p-1)}$, then

$$
\omega_{m}(x)=\left[\frac{\Gamma\left(q_{1}\right) \cdots \Gamma\left(q_{m}\right)}{\Gamma\left(q_{1}+\cdots+q_{m}\right)}\right]^{p-1} x^{\left(q_{1}+\cdots+q_{m}-1\right)(p-1)} .
$$

On the other hand, for $p=1, q_{j} \geq 0$ and $v_{j}(t)=t^{q_{j}}$, a simple calculation yields

$$
\omega_{m}(x)=\frac{q_{1}^{q_{1}} \cdots q_{m}^{q_{m}}}{\left(q_{1}+\cdots+q_{m}\right)^{q_{1}+\cdots+q_{m}}} x^{q_{1}+\cdots+q_{m}}
$$

where $q^{q}$ is taken to be 1 whenever $q=0$. Thus, we obtain the following result for power weights on $R^{+}$.

Corollary 1. If $q_{j} \geq 0, j=1, \ldots, m$, then

$$
\begin{aligned}
\int_{R^{+}} x^{-\left(q_{1}+\cdots+q_{m}\right)} \mid\left[\prod_{j=1}^{m} * F_{j}\right] & (x) \mid d x \\
\leq & \left.\leq \frac{q_{1}^{q_{1}} \cdots q_{m}^{q_{m}}}{\left(q_{1}+\cdots+q_{m}\right)^{q_{1}+\cdots+q_{m}}}\right] \prod_{j=1}^{m} \int_{R^{+}}\left|F_{j}(t)\right| t^{-q_{j}} d t
\end{aligned}
$$

for all complex valued measurable functions $F_{j}$ with $\int_{R^{+}}\left|F_{j}(t)\right| t^{-q_{j}} d t<\infty, j=$ $1, \ldots, m$. 
If $1<p<\infty$ and $q_{j}>0, j=1, \ldots, m$, then

$$
\begin{aligned}
& \int_{R^{+}} x^{-\left(q_{1}+\cdots+q_{m}-1\right)(p-1)}\left|\left[\prod_{j=1}^{m} * F_{j}\right](x)\right|^{p} d x \\
& \leq\left[\frac{\Gamma\left(q_{1}\right) \cdots \Gamma\left(q_{m}\right)}{\Gamma\left(q_{1}+\cdots+q_{m}\right)}\right]^{p-1} \prod_{j=1}^{m} \int_{R^{+}}\left|F_{j}(t)\right|^{p} t^{\left(1-q_{j}\right)(p-1)} d t
\end{aligned}
$$

for all complex valued measurable functions $F_{j}$ with $\int_{R^{+}}\left|F_{j}(t)\right|^{p} t^{\left(1-q_{j}\right)(p-1)} d t<\infty$, $j=1, \ldots, m$. Unless $F_{j}=0$ a.e. for some $j$, equality holds in (1.4) if and only if $F_{j}(t)=c_{j} e^{-\alpha t+i \beta t} t^{q_{j}-1}$ a.e. for complex constants $c_{j} \neq 0, j=1, \ldots, m$, and real constants $\alpha, \beta$ with $\alpha>0$.

Special cases of Corollary 1 are known. The case $p=2, q_{j}=1$ for all $j$, and $m$ even was obtained by Saitoh [4] and this was extended to $q_{j}>0$ and arbitrary $m$ by Burbea [1]. For $1<p<\infty$ Cwikel and Kerman [2] obtained Corollary 1 for the case that $q_{j}=1$ for each $j$.

The analogue of Theorem 1 for $R^{n}$ is as follows. As usual, for $x=\left(x_{1}, \ldots, x_{n}\right)$ and $y=\left(y_{1}, \ldots, y_{n}\right)$ we write $x \cdot y=x_{1} y_{1}+\cdots+x_{n} y_{n}$.

Theorem 2. Let $1 \leq p<\infty, 1 / p+1 / p^{\prime}=1$, and let $m \geq 2$ be an integer. Suppose $v_{j}, j=1, \ldots, m$, are nonnegative measurable functions on $R^{n}$ with $v_{j}^{1 / p} \in L^{p^{\prime}}\left(R^{n}\right)$. Define $\omega_{1}=v_{1}$, and set

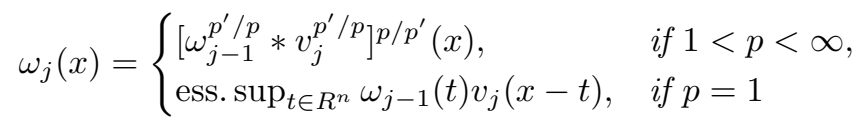

for $j=2, \ldots, m$. Then

$$
\int_{R^{n}}\left|\left[\prod_{j=1}^{m} * F_{j}\right](x)\right|^{p} \frac{d x}{\omega_{m}(x)} \leq \prod_{j=1}^{m} \int_{R^{n}}\left|F_{j}(t)\right|^{p} \frac{d t}{v_{j}(t)}
$$

for all complex valued measurable functions $F_{j}$ with $\int_{R^{n}}\left|F_{j}(t)\right|^{p} \frac{d t}{v_{j}(t)}<\infty, j=$ $1, \ldots, m$.

If $1<p<\infty$, equality holds in (1.5) if

$$
F_{j}(t)=c_{j} e^{\alpha \cdot t+i \beta \cdot t} v_{j}^{p^{\prime} / p}(t) \quad \text { a.e. }
$$

for complex constants $c_{j}$ and fixed $\alpha, \beta \in R^{n}$ such that $\int_{R^{n}} e^{p(\alpha \cdot t)} v_{j}^{p^{\prime} / p}(t) d t<\infty$, $j=1, \ldots, m$. Conversely, if $v_{j}(t)>0$ on an open path connected set $I_{j}$ and $v_{j}(t)=0$ on $R^{n} \backslash I_{j}, j=1, \ldots, m$, and equality holds in $(1.5)$, then $F_{j}, j=1, \ldots, m$, is given by (1.6) unless $F_{j}(t)=0$ a.e. for some $j$.

For $n=1$ and $v_{1}(t)=v_{2}(t)=\chi_{\{t:|t|<r\}}(t)$ for fixed $r>0$ we have $\omega_{2}(x)=$ $(2 r-|x|)^{p-1} \chi_{\{x:|x|<2 r\}}(x)$. Thus we obtain the following result. The case $p=2$ was obtained by Saitoh [5, p. 54] (note that the result is misstated there).

Corollary 2. If $1 \leq p<\infty, F_{1}$ and $F_{2}$ belong to $L^{p}(R)$ and are supported in the interval $\{t:|t|<r\}$, then

$$
\int_{\{x:|x|<2 r\}} \frac{\left|\left(F_{1} * F_{2}\right)(x)\right|^{p}}{(2 r-|x|)^{p-1}} d x \leq \int_{\{t:|t|<r\}}\left|F_{1}(t)\right|^{p} d t \int_{\{t:|t|<r\}}\left|F_{2}(t)\right|^{p} d t .
$$


The Poisson kernel $P_{b}(t)$ given by

$$
P_{b}(t)=\frac{1}{\pi} \frac{b}{\left[|t|^{2}+b^{2}\right]^{(n+1) / 2}}, \quad t \in R^{n}, b>0,
$$

satisfies $P_{b}(a t)=a^{-n} P_{b / a}(t)$ and the semigroup property $P_{b_{1}} * P_{b_{2}}=P_{b_{1}+b_{2}}$. Thus, taking $v_{j}(t)=\left[P_{b_{j}}\left(a_{j} t\right)\right]^{p / p^{\prime}}$ for $1<p<\infty, a_{j}>0, b_{j}>0$ yields

$$
\omega_{m}^{p^{\prime} / p}(x)=\frac{\left(\prod_{j=1}^{m} a_{j}\right)^{-n} \sum_{j=1}^{m} \frac{b_{j}}{a_{j}}}{\pi\left[|x|^{2}+\left(\sum_{j=1}^{m} \frac{b_{j}}{a_{j}}\right)^{2}\right]^{(n+1) / 2}}
$$

and we have the following corollary which was obtained in the case $n=1, m=p=2$ by Saitoh [6, p. 516].

Corollary 3. If $1<p<\infty, a_{j}>0$ and $b_{j}>0$ for $j=1, \ldots, m$, then

$$
\begin{aligned}
\int_{R^{n}} \mid & {\left.\left[\prod_{j=1}^{m} * F_{j}\right](x)\right|^{p}\left[\left(\prod_{j=1}^{m} a_{j}\right)^{2}|x|^{2}+\left(\prod_{j=1}^{m} a_{j} \sum_{j=1}^{m} \frac{b_{j}}{a_{j}}\right)^{2}\right]^{(n+1)(p-1) / 2} d x } \\
& \leq\left[\pi^{m-1}\left(\prod_{j=1}^{m} \frac{a_{j}}{b_{j}}\right) \sum_{j=1}^{m} \frac{b_{j}}{a_{j}}\right]^{p-1} \prod_{j=1}^{m} \int_{R^{n}}\left|F_{j}(t)\right|^{p}\left[a_{j}^{2}|t|^{2}+b_{j}^{2}\right]^{(n+1)(p-1) / 2} d t
\end{aligned}
$$

for all complex valued functions $F_{j}$ satisfying

$$
\int_{R^{n}}\left|F_{j}(t)\right|^{p}\left[a_{j}^{2}|t|^{2}+b_{j}^{2}\right]^{(n+1)(p-1) / 2} d t<\infty, \quad j=1, \ldots, m .
$$

Unless $F_{j}=0$ a.e. for some $j$, equality holds if and only if

$$
F_{j}(t)=c_{j} e^{i \beta \cdot t}\left[a_{j}^{2}|t|^{2}+b_{j}^{2}\right]^{-(n+1) / 2} \quad \text { a.e. }
$$

for complex constants $c_{j} \neq 0, j=1, \ldots, m$, and fixed $\beta \in R^{n}$.

For power weights on $R^{n}$ we have the following result, where we have set $\gamma(\alpha)=$ $\pi^{n / 2} 2^{\alpha} \Gamma(\alpha / 2) / \Gamma((n-\alpha) / 2)$ for $0<\alpha<n$.

Corollary 4. If $1<p<\infty, m \geq 2$ an integer and $q_{j}>0$ with $\sum_{j=1}^{m} q_{j}<n$, then

$$
\begin{aligned}
\int_{R^{n}}|x|^{-\left(q_{1}+\cdots+q_{m}-n\right)(p-1)}\left|\left[\prod_{j=1}^{m} * F_{j}\right](x)\right|^{p} d x & \\
\leq & {\left[\frac{\gamma\left(q_{1}\right) \cdots \gamma\left(q_{m}\right)}{\gamma\left(q_{1}+\cdots+q_{m}\right)}\right]^{p-1} \prod_{j=1}^{m} \int_{R^{n}}\left|F_{j}(t)\right|^{p}|t|^{\left(n-q_{j}\right)(p-1)} d t }
\end{aligned}
$$

for all complex valued measurable functions $F_{j}$ with $\int_{R^{n}}\left|F_{j}(t)\right|^{p} t^{\left(n-q_{j}\right)(p-1)} d t<\infty$, $j=1, \ldots, m$. Equality holds if and only if $F_{j}(t)=0$ a.e. for some $j$.

The convolution defined on $R^{+}$by $\left(f_{1} \star f_{2}\right)(x)=\int_{R^{+}} f_{1}(t) f_{2}(x / t) d t / t$ may be expressed as $\left(F_{1} * F_{2}\right)(\xi)$ where $x=e^{\xi}$ and $F_{j}(\xi)=f_{j}(x), j=1,2$. Thus Theorem 2 yields the following corollary. 
Corollary 5. Let $1 \leq p<\infty, 1 / p+1 / p^{\prime}=1$, and let $m \geq 2$ be an integer. Suppose $v_{j}, j=1, \ldots, m$, are nonnegative measurable functions on $R^{+}$with $v_{j}^{1 / p} \in$ $L^{p^{\prime}}\left(R^{+}, d t / t\right)$. Define $\omega_{1}=v_{1}$, and set

$$
\omega_{j}(x)= \begin{cases}{\left[\omega_{j-1}^{p^{\prime} / p} \star v_{j}^{p^{\prime} / p}\right]^{p / p^{\prime}}(x),} & \text { if } 1<p<\infty, \\ \operatorname{ess}^{\sup _{t \in R^{+}}} \omega_{j-1}(t) v_{j}(x / t), & \text { if } p=1\end{cases}
$$

for $j=2, \ldots, m$. Then

$$
\int_{R^{+}}\left|\left[\prod_{j=1}^{m} \star f_{j}\right](x)\right|^{p} \frac{d x}{x \omega_{m}(x)} \leq \prod_{j=1}^{m} \int_{R^{+}}\left|f_{j}(t)\right|^{p} \frac{d t}{t v_{j}(t)}
$$

for all complex valued measurable functions $f_{j}$ with $\int_{R^{+}}\left|f_{j}(t)\right|^{p} \frac{d t}{t v_{j}(t)}<\infty, j=$ $1, \ldots, m$.

If $1<p<\infty$, equality holds in (1.7) if

$$
f_{j}(t)=c_{j} t^{\alpha+i \beta} v_{j}^{p^{\prime} / p}(t) \quad \text { a.e. }
$$

for complex constants $c_{j}$ and real numbers $\alpha, \beta$ such that $\int_{R^{+}} t^{p \alpha} v_{j}^{p^{\prime} / p}(t) d t / t<\infty$, $j=1, \ldots, m$. Conversely, if $v_{j}(t)>0$ on an open interval $I_{j}$ and $v_{j}(t)=0$ on $R^{+} \backslash I_{j}, j=1, \ldots, m$, and equality holds in (1.7), then $f_{j}, j=1, \ldots, m$, is given by $(1.8)$ unless $f_{j}(t)=0$ a.e. for some $j$.

\section{Proofs}

We write $|E|$ for the Lebesgue measure of a measurable $E \subset R^{n}, E_{1}+E_{2}=$ $\left\{t_{1}+t_{2}: t_{1} \in E_{1}, t_{2} \in E_{2}\right\}$ for $E_{1}, E_{2} \subset R^{n}$ and $r E=\{r t: t \in E\}$ for $E \subset R^{n}$ and $r>0$.

We will need the following Lemma.

Lemma. Let $I_{j} \subset R^{n}$ be open and path connected and suppose $f_{j}$ is complex valued and measurable on $I_{j}, j=1,2$. If $h$ is measurable on $I_{1}+I_{2}$ and satisfies

$$
f_{1}\left(x_{1}\right) f_{2}\left(x_{2}\right)=h\left(x_{1}+x_{2}\right) \quad \text { a.e. } \quad x_{1} \in I_{1}, \quad x_{2} \in I_{2},
$$

then there are $\alpha, \beta \in R^{n}$ and complex constants $c_{j} \neq 0$ such that

$$
f_{j}(t)=c_{j} e^{\alpha \cdot t+i \beta \cdot t} \quad \text { a.e. } t \in I_{j}, \quad j=1,2,
$$

unless $f_{j}=0$ a.e. on $I_{j}$ for some $j$.

Proof of Lemma. It suffices to prove the Lemma for the case that $I_{1}$ and $I_{2}$ are open balls of the same radius. For if this has been proved, then if $f_{j}$ is not zero almost everywhere on $I_{j}, j=1,2$, there are balls $B_{j} \subset I_{j}$ of the same radius $r$ such that $f_{j}$ is not zero almost everywhere on $B_{j}$ and hence

$$
f_{j}(t)=c_{j} e^{\alpha \cdot t+i \beta \cdot t} \quad \text { a.e. } \quad t \in B_{j}, \quad j=1,2 .
$$

But then, since $I_{1}$ is open and path connected, if $x \in I_{1} \backslash B_{1}$ there is a finite sequence of overlapping balls $B_{k}, k=3, \ldots, m$, of equal radius not exceeding $r$ such that $\left|B_{1} \cap B_{3}\right|>0,\left|B_{k} \cap B_{k+1}\right|>0, k=3, \ldots, m-1$, and $x \in B_{m}$. Choosing a ball $B_{2}^{\prime} \subset B_{2}$ with radius equal to that of $B_{3}$ and noting that $(2.2)$ shows $\left|f_{1}\right|>0$ a.e. on $B_{3} \cap B_{1}$ and $\left|f_{2}\right|>0$ a.e. on $B_{2}^{\prime}$, the special case of the Lemma applied successively to the balls $B_{k} \subset I_{1}, k=3, \ldots, m, B_{2}^{\prime} \subset I_{2}$ shows that $f_{1}(t)=c_{1} e^{\alpha \cdot t+i \beta \cdot t}$ a.e. on 
$B_{m}$. Thus $f_{1}(t)=c_{1} e^{\alpha \cdot t+i \beta \cdot t}$ a.e. on a neighbourhood of $x$ and hence also a.e. on $I_{1}$. A similar argument shows that $f_{2}(t)=c_{2} e^{\alpha \cdot t+i \beta \cdot t}$ a.e. on $I_{2}$.

Suppose then that $I_{1}$ and $I_{2}$ are balls of the same radius, and that $f_{j}$ is not zero almost everywhere on $I_{j}, j=1,2$. By considering $F_{j}\left(x_{j}\right)=f_{j}\left(x_{j}-a_{j}\right)$ and $H(x)=h\left(x-a_{1}-a_{2}\right)$ if necessary, we may further assume that $I_{1}=I_{2}=B$ where $B$ has center at the origin.

We first prove that $\left|f_{j}\right|>0$ a.e. on $B, j=1,2$. Let $A_{j}=\left\{x \in B: f_{j}(x)=0\right\}$ and suppose, to derive a contradiction, that $\left|A_{1}\right|+\left|A_{2}\right|>0$. If $\left|A_{1}\right|>0$, then (2.1) and Fubini's Theorem (for nonnegative but not necessarily integrable functions) shows that

$$
\begin{aligned}
0=\int_{A_{1}}\left|f_{1}\left(x_{1}\right)\right| d x_{1} \int_{B}\left|f_{2}\left(x_{2}\right)\right| d x_{2} & =\iint_{A_{1} \times B}\left|h\left(x_{1}+x_{2}\right)\right| d x_{1} d x_{2} \\
& =\int_{A_{1}} d x_{1} \int_{B}\left|h\left(x_{1}+x_{2}\right)\right| d x_{2}
\end{aligned}
$$

and hence, for almost all $x_{1} \in A_{1}, h(t)=0$ a.e. $t \in x_{1}+B$. A similar argument holds with the role of $f_{1}$ and $f_{2}$ reversed. Thus, for a.e. $x \in A_{1} \cup A_{2}$,

$$
h(t)=0 \quad \text { a.e. } \quad t \in x+B
$$

and in particular, since $\left|A_{1} \cup A_{2}\right|>0$, there is $x_{0} \in B$ such that $h=0$ a.e. on $x_{0}+r_{0} B$ with $r_{0}=1$. For $m \geq 0$, set $x_{m+1}=x_{m} / 2$ and $r_{m+1}=r_{m} / 2+1$. Now, suppose it has been verified that $h=0$ a.e. on $x_{m}+r_{m} B$ for some $m$, and let $E=x_{m} / 2+\left(r_{m} / 2\right) B$. Then $E+E=x_{m}+r_{m} B$ and hence (2.1) shows

$$
\int_{E}\left|f_{1}\left(x_{1}\right)\right| d x_{1} \int_{E}\left|f_{2}\left(x_{2}\right)\right| d x_{2}=\iint_{E \times E}\left|h\left(x_{1}+x_{2}\right)\right| d x_{1} d x_{2}=0
$$

so that, for some $j, f_{j}=0$ a.e. on $E$. Hence (2.3) shows that $h=0$ a.e. on $E+B=x_{m+1}+r_{m+1} B$. Thus $h=0$ a.e. on $x_{m}+r_{m} B$ for all $m \geq 0$. Clearly, $x_{m} \rightarrow 0$ and an easy induction shows that $r_{m} \uparrow 2$. Thus, $h=0$ a.e. on $2 B$, and taking $E=B$ in (2.4) we find $f_{j}=0$ a.e. on $B$ for some $j$, the desired contradiction.

Now, since $\left|f_{j}\right|>0$ a.e. on $B, j=1,2,(2.1)$ shows that

$$
\frac{f_{2}\left(x_{2}\right)}{f_{1}\left(x_{2}\right)}=\frac{f_{2}\left(x_{1}\right)}{f_{1}\left(x_{1}\right)} \quad \text { a.e. } \quad x_{j} \in B
$$

and hence $f_{2}=c f_{1}$ a.e. on $B$ for a complex constant $c \neq 0$, and hence also

$$
f_{j}\left(x_{1}\right) f_{j}\left(x_{2}\right)=f_{j}^{2}\left(\frac{x_{1}+x_{2}}{2}\right) \quad \text { a.e. } \quad x_{j} \in B, \quad j=1,2 .
$$

Writing $f_{j}(t)=e^{i \theta_{j}(t)}\left|f_{j}(t)\right|$ where $\theta_{j}(t) \in(-\pi, \pi],(2.5)$ shows that $\log \left|f_{j}(t)\right|$ and $\theta_{j}(t), j=1,2$, satisfy the functional equation

$$
\phi\left(\frac{x_{1}+x_{2}}{2}\right)=\frac{\phi\left(x_{1}\right)+\phi\left(x_{2}\right)}{2} \quad \text { a.e. } \quad x_{j} \in B .
$$

Since the solutions of this equation [7, p. 231] are of the form $\phi(t)=\alpha \cdot t+\gamma$ a.e. for fixed $\alpha \in R^{n}$ and $\gamma \in R$, this shows that there are $\alpha, \beta \in R^{n}$ and complex constants $c_{j} \neq 0, j=1,2$, such that $f_{j}(t)=c_{j} e^{\alpha \cdot t+i \beta \cdot t}$ a.e. on $B$. This completes the proof of the Lemma. 
Proof of Theorem 1. Observe first that $\omega_{j}$ is well defined, nonnegative, and $\omega_{j}^{1 / p} \in L^{p^{\prime}}(0, R)$ for every $R>0$. Thus $\omega_{j}$ satisfies the same hypothesis satisfied by each weight function $v_{j}$. For $j=2$ this follows from

$$
\begin{aligned}
\int_{0}^{R} \omega_{2}^{p^{\prime} / p}(x) d x & =\int_{0}^{R} d x \int_{0}^{x} v_{1}^{p^{\prime} / p}(t) v_{2}^{p^{\prime} / p}(x-t) d t \\
& =\int_{0}^{R} v_{1}^{p^{\prime} / p}(t) d t \int_{t}^{R} v_{2}^{p^{\prime} / p}(x-t) d x \\
& \leq \int_{0}^{R} v_{1}^{p^{\prime} / p}(t) d t \int_{0}^{R} v_{2}^{p^{\prime} / p}(x) d x
\end{aligned}
$$

if $1<p<\infty$ and from

$$
\begin{aligned}
\underset{0<x<R}{\text { ess. } \sup } w_{2}(x) & =\underset{0<x<R}{\operatorname{ess} . \sup } \underset{0<t<x}{\operatorname{ess} . \sup } v_{1}(t) v_{2}(x-t) \\
\leq & \underset{0<t<R}{\operatorname{ess} . \sup } v_{1}(t) \underset{0<x<\sup _{0}}{\operatorname{ess}} v_{2}(x)
\end{aligned}
$$

if $p=1$, and an induction yields the same conclusion for $j>2$.

Now let $E_{j}=\left\{t: v_{j}(t)>0\right\}$ and $G_{j}=\left\{t: \omega_{j}(t)>0\right\}$. We prove (1.2) by induction. With $\prod_{j=1}^{1} * F_{j}=F_{1}$, (1.2) holds for $m=1$, so we suppose that $k \geq 2, \int_{R^{+}}\left|F_{j}(t)\right|^{p} d t / v_{j}(t)<\infty, j=1, \ldots, k$, and that (1.2) has been verified for $m=k-1$. Set $H_{k-1}(t)=\left[\prod_{j=1}^{k-1} * F_{j}\right](t)$. Then $\int_{R^{+}}\left|H_{k-1}(t)\right|^{p} d t / \omega_{k-1}(t)<\infty$ shows that $H_{k-1}=0$ a.e. on $R^{+} \backslash G_{k-1}$ so that

$$
\begin{aligned}
& \left|\int_{0}^{x} H_{k-1}(t) F_{k}(x-t) d t\right|^{p} \\
& \quad=\left|\int_{0}^{x} \frac{H_{k-1}(t) \chi_{G_{k-1}}(t)}{\omega_{k-1}^{1 / p}(t)} \frac{F_{k}(x-t) \chi_{E_{k}}(x-t)}{v_{k}^{1 / p}(x-t)}\left[\omega_{k-1}(t) v_{k}(x-t)\right]^{1 / p} d t\right|^{p} \\
& \quad \leq\left[\int_{0}^{x} \frac{\left|H_{k-1}(t)\right|^{p}}{\omega_{k-1}(t)} \frac{\left|F_{k}(x-t)\right|^{p}}{v_{k}(x-t)} d t\right] \omega_{k}(x)
\end{aligned}
$$

by Hölder's inequality. Fubini's Theorem shows that the last integral is finite a.e. since

$$
\begin{aligned}
\int_{R^{+}} & \int_{0}^{x} \frac{\left|H_{k-1}(t)\right|^{p}}{\omega_{k-1}(t)} \frac{\left|F_{k}(x-t)\right|^{p}}{v_{k}(x-t)} d t d x \\
& =\int_{R^{+}} \int_{t}^{\infty} \frac{\left|H_{k-1}(t)\right|^{p}}{\omega_{k-1}(t)} \frac{\left|F_{k}(x-t)\right|^{p}}{v_{k}(x-t)} d x d t \\
& =\int_{R^{+}}\left|H_{k-1}(t)\right|^{p} \frac{d t}{\omega_{k-1}(t)} \int_{R^{+}}\left|F_{k}(x)\right|^{p} \frac{d x}{v_{k}(x)}<\infty .
\end{aligned}
$$

Thus, (2.6) shows that $\left(H_{k-1} * F_{k}\right)(x)$ exists a.e. and $H_{k-1} * F_{k}=0$ a.e. on $R^{+} \backslash G_{k}$. Combining (2.6) and (2.7) yields

$$
\int_{R^{+}}\left|\left[\prod_{j=1}^{k} * F_{j}\right](x)\right|^{p} \frac{d x}{\omega_{k}(x)} \leq\left[\prod_{j=1}^{k-1} \int_{R^{+}}\left|F_{j}(t)\right|^{p} \frac{d t}{v_{j}(t)}\right]\left[\int_{R^{+}}\left|F_{k}(t)\right|^{p} \frac{d t}{v_{k}(t)}\right]
$$

which is (1.2) for $m=k$. This completes the induction proof of (1.2).

If $1<p<\infty$, a straightforward calculation shows that equality holds in (1.2) if $F_{j}$ is given by (1.3). Conversely, suppose $E_{j}=I_{j}$ is an open interval and equality holds in (1.2). A simple induction shows that $G_{j}=G_{j-1}+I_{j}$ and hence $G_{j}$ is 
also an open interval. It follows from (2.6) and the induction argument that, apart from the cases in which $F_{j}=0$ a.e. for some $j$, equality in (1.2) requires equality in Hölder's inequality for each $k, k=2, \ldots, m$. Thus [3, p. 39] there is a complex valued function $h_{k}$ such that for almost all $x \in R^{+}$

$$
H_{k-1}(t) F_{k}(x-t)=h_{k}(x) \omega_{k-1}^{p^{\prime} / p}(t) v_{k}^{p^{\prime} / p}(x-t) \quad \text { a.e. } \quad t \in G_{k-1} \cap\left(x-I_{k}\right)
$$

which we write as

$$
\text { for a.e. } x \in R^{+}, \quad f_{k 1}(t) f_{k 2}(x-t)=h_{k}(x) \quad \text { a.e. } \quad t \in G_{k-1} \cap\left(x-I_{k}\right),
$$

where

$$
\begin{aligned}
& f_{k 1}(t)=H_{k-1}(t) \omega_{k-1}^{-p^{\prime} / p}(t), \quad t \in G_{k-1}, \\
& f_{k 2}(t)=F_{k}(t) v_{k}^{-p^{\prime} / p}(t), \quad t \in I_{k} .
\end{aligned}
$$

We first show that $h_{k}$ is measurable on $G_{k}$. Let $U(x)$ be positive and integrable on $R^{+}$, say $U(x)=e^{-x}$, and let $V(t)$ be real valued, continuous, bounded and strictly increasing on $R$, say $V(t)=\arctan t$. Then

$\Psi(x, t)=\frac{U(x) \chi_{G_{k}}(x)}{\omega_{k}^{p^{\prime} / p}(x)} V\left(\operatorname{Re}\left[f_{k 1}(t) f_{k 2}(x-t)\right]\right) \omega_{k-1}^{p^{\prime} / p}(t) \chi_{G_{k-1}}(t) v_{k}^{p^{\prime} / p}(x-t) \chi_{I_{k}}(x-t)$ is integrable on $R^{+} \times R^{+}$and hence Fubini's Theorem shows that

$$
\Psi(x)=\int_{R^{+}} \Psi(x, t) d t
$$

is measurable. In view of (2.8), $\Psi(x)=U(x) V\left(\operatorname{Re}\left[h_{k}(x)\right]\right) \chi_{G_{k}}(x)$ for a.e. $x \in R^{+}$ and hence $\operatorname{Re}\left[h_{k}(x)\right]=V^{-1}(\Psi(x) / U(x))$ a.e. on $G_{k}$. Thus, $\operatorname{Re}\left[h_{k}\right]$ is measurable on $G_{k}$. A similar argument applies to $\operatorname{Im}\left[h_{k}(x)\right]$, and hence $h_{k}$ is measurable on $G_{k}$.

Since $H_{k}$ is measurable on $G_{k}, \phi\left(x_{1}, x\right)=\left|f_{k 1}\left(x_{1}\right) f_{k 2}\left(x-x_{1}\right)-h_{k}(x)\right| \chi_{I_{k}}\left(x-x_{1}\right)$ is measurable for $\left(x_{1}, x\right) \in G_{k-1} \times G_{k}$ and in view of (2.8) and Fubini's Theorem we have

$$
\begin{aligned}
0 & =\int_{G_{k}} d x \int_{G_{k-1}} \phi\left(x_{1}, x\right) d x_{1}=\iint_{G_{k} \times G_{k-1}} \phi\left(x_{1}, x\right) d x d x_{1} \\
& =\int_{G_{k-1}} d x_{1} \int_{G_{k}} \phi\left(x_{1}, x\right) d x \\
& =\int_{G_{k-1}} d x_{1} \int_{\left[G_{k-1}+I_{k}\right]-x_{1}}\left|f_{k 1}\left(x_{1}\right) f_{k 2}\left(x_{2}\right)-h_{k}\left(x_{2}+x_{1}\right)\right| \chi_{I_{k}}\left(x_{2}\right) d x_{2} .
\end{aligned}
$$

Since $x_{1} \in G_{k-1}$ implies $I_{k} \subset\left[G_{k-1}+I_{k}\right]-x_{1}$, this shows that

$$
f_{k 1}\left(x_{1}\right) f_{k 2}\left(x_{2}\right)=h_{k}\left(x_{1}+x_{2}\right) \quad \text { a.e. } \quad x_{1} \in G_{k-1}, \quad x_{2} \in I_{k} .
$$

Thus, the Lemma now shows that (2.8) requires

$$
f_{k j}\left(x_{j}\right)=c_{j} e^{\alpha_{k} x_{j}+i \beta_{k} x_{j}} \quad \text { a.e. } \quad x_{1} \in G_{k-1}, \quad x_{2} \in I_{k}
$$

for real constants $\alpha_{k}, \beta_{k}$ and complex constants $c_{j} \neq 0, j=1,2$, unless $f_{k j}=0$ a.e. for some $j$.

The necessity of (1.3) may now be proved by induction. If $F_{j}=0$ a.e. fails for each $j, j=1, \ldots, m$, then taking $k=2$ in (2.8) shows, in view of (2.9), that (1.3) holds for $j=1,2$, and to complete the induction, suppose (1.3) has been verified 
for $j=1, \ldots, k-1$. Then $H_{k-1}(t)=\left[\prod_{j=1}^{k-1} * F_{j}\right](t)=\left(\prod_{j=1}^{k-1} c_{j}\right) e^{\alpha t+i \beta t} \omega_{k-1}^{p^{\prime} / p}(t)$ a.e. on $G_{k-1}$ and hence (2.8) and (2.9) show that $F_{k}(t)=c_{k} e^{\alpha t+i \beta t} v_{k}^{p^{\prime} / p}(t)$ a.e. on $I_{k}$. Thus (1.3) holds for $j=k$, and the proof is complete.

Proof of Theorem 2 and Corollary 4. The proof of Theorem 2 is similar to that of Theorem 1 so the details are omitted. Note however that the local integrability hypothesis imposed on $v_{j}$ in Theorem 1 was used only to ensure that the $\omega_{j}$ are well defined and satisfy the same hypothesis as the weights $v_{j}$. In $R^{n}$, local integrability is not sufficient to provide this assurance, and hence the stronger hypothesis of Theorem 2.

On the other hand, any hypothesis ensuring that the $\omega_{j}$ are well defined is sufficient to validate the remaining arguments used in the proof. Thus, while the power functions $v_{j}(t)=|t|^{\left(q_{j}-n\right)(p-1)}$ do not satisfy the hypothesis of Theorem 2, the corresponding $\omega_{j}$ are well defined under the hypothesis of Corollary 4 as we now show.

For $x \neq 0$ and $e_{1}=(1,0, \ldots, 0) \in R^{n}$,

$$
\begin{aligned}
\left(|t|^{q_{1}-n} *|t|^{q_{2}-n}\right)(x) & =|x|^{q_{1}+q_{2}-n} \int_{R^{n}}|t|^{q_{1}-n}\left|e_{1}-t\right|^{q_{2}-n} d t \\
& =B_{n}\left(q_{1}, q_{2}\right)|x|^{q_{1}+q_{2}-n}
\end{aligned}
$$

and the Beta integral $B_{n}\left(q_{1}, q_{2}\right)$ is finite if $\min \left[q_{1}, q_{2}\right]>0$ and $q_{1}+q_{2}<n$ with value $\gamma\left(q_{1}\right) \gamma\left(q_{2}\right) / \gamma\left(q_{1}+q_{2}\right)$ where

$$
\gamma(\alpha)=\pi^{n / 2} 2^{\alpha} \Gamma(\alpha / 2) / \Gamma((n-\alpha) / 2) .
$$

Thus, with $v_{j}=|t|^{\left(q_{j}-n\right)(p-1)}, q_{j}>0$ and $q_{1}+\cdots+q_{m}<n$, an induction shows we have

$$
\omega_{m}(x)=\left[\frac{\gamma\left(q_{1}\right) \cdots \gamma\left(q_{m}\right)}{\gamma\left(q_{1}+\cdots+q_{m}\right)}\right]^{p-1}|x|^{\left(q_{1}+\cdots+q_{m}-n\right)(p-1)} .
$$

Thus, in view of the remarks above, we have the inequality of Corollary 4 , and since $\int_{R^{n}} e^{p(\alpha \cdot t)}|t|^{\left(q_{j}-n\right)(p-1)} d t=\infty$ for every $\alpha \in R^{n}$, equality requires $F_{j}=0$ a.e. for some $j$.

\section{REFERENCES}

[1] J. Burbea, Inequalities for weighted $L^{2}$-functions on the half-line, Arch. Math. 47 (1986), pp. 427-437. MR 88c:30015

[2] M. Cwikel and R. Kerman, On a convolution inequality of Saitoh, Proc. Amer. Math. Soc. 124 (1996), pp. 773-777. MR 96g:26027

[3] E. Lieb and M. Loss, Analysis, Graduate Studies in Mathematics, vol. 14, Amer. Math. Soc., Providence, RI, 1996. MR 98b:00004

[4] S. Saitoh, A fundamental inequality in the convolution of $L_{2}$ functions on the half line, Proc. Amer. Math. Soc. 91 (1984), pp. 285-286. MR 85j:30010

[5] - On the convolution of $L_{2}$ functions, Kodai Math. J. 9 (1986), pp. 50-57. MR 87e: 42016

[6] Inequalities in the most simple Sobolev space and convolutions of $L_{2}$ functions with weights, Proc. Amer. Math. Soc. 118 (1993), pp. 515-520. MR 93g:46029

[7] J. Tabor, Cauchy and Jensen equations in a restricted domain almost everywhere, Publ. Math. Debrecen 39 (1991), pp. 219-235. MR 93b:39007

Department of Mathematical Sciences, University of Alberta, Edmonton, Alberta, Canada T6G 2G1

E-mail address: kanderse@vega.math.ualberta.ca 